\title{
Chromatin remodelers as active Brownian dimers
}

\author{
R. Blossey ${ }^{1}$ and H. Schiessel ${ }^{2}$ \\ ${ }^{1}$ University of Lille, UGSF CNRS UMR8576, 59000 Lille, France. \\ ${ }^{2}$ Institute Lorentz for Theoretical Physics, Leiden University, Niels Bohrweg 2, 2333 \\ CA Leiden, The Netherlands. \\ E-mail: ralf.blossey@univ-lille.fr
}

\begin{abstract}
Chromatin remodelers are molecular motors which actively displace nucleosomes on chromatin. Recent results on the structural properties of these motors indicate that the displacement of nucleosomal DNA corresponds to an inchworm motion induced by the generation and propagation of twist defects. Here we show that this basic action mechanism can be described by a coarse-grained active Brownian dimer (ABD) model, thereby quantitatively rationalizing the notion of inchworm motion. The model allows for extensions to more microscopic as well towards more macroscopic descriptions of chromatin hydrodynamics.
\end{abstract}

Keywords: nucleosome, chromatin remodeler, helicase, active Brownian dimer

Submitted to: J. Phys. A: Math. Gen. 
Introduction. Active systems currently are one of the most intensive fields of research within the statistical physics community. Built on a large body of work dealing with individual motors (see, e.g. [1]), the field has turned towards studies of the collective behaviour of 'active' constituents, see, e.g. [2]. Here we are concerned with chromatin remodeling motors which actively displace and remove nucleosomes from the chromatin fiber, which have so far received only little attention in the statistical physics or biophysics literature [1]. The number of modeling attempts of individual remodeler dynamics has been rather limited so far; see, e.g., the references $[3,4,5,6]$; but also the collective behaviour of chromatin remodelers is beginning to attract attention $[7,8,9,10]$.

This lack of attention may in part be explained by the structural complexity and size of remodelers which has so far allowed to resolve only few and in particular often partial structures; this also impeded studies of remodeler dynamics, except in artificial constructs (see below). Very recently, the more widespread use of cryoelectron microscopy and FRET-imaging techniques have led to numerous new results, in particular on the (small) chromatin remodeler Chd1 [11, 12, 13, 14, 15].

Chromatin remodelers are built around evolutionarily conserved two-domain ATPase units which belong to the helicase-related superfamily II (SF2), and can be grouped into a small number of families which differ from each other by their accessory subunits [16]. These molecular motors play crucial rules in numerous chromatin-based processes such as the activation or the repression of transcription and DNA repair $[17,18,19]$; their biological relevance is underscored by the recent understanding that remodeler dysfunction is one source of regulatory diseases such as cancer [20]. A better understanding of how the motors act on the nucleosomes is thus of key relevance.

In this paper we show that, at a coarse-grained level, the DNA displacement around the nucleosome of a stripped-down version of chromatin remodeling enzymes can be very well captured in terms of a different type of model, an active Brownian dimer model. Such models were developed several years ago in the context of the simpler linear motors $[21]$.

Mapping of a chromatin remodeler to an active Brownian dimer. Our mapping relies on recent structural biology results which we now briefly review. Liu et al. [22, 23] have described the structure of a truncated version of a basic remodeler which they call ScSnf2 in complex with a nucleosome core-particle with a 167-bp DNA fragment containing the '601' positioning sequence [24]. Cryo-electron microscopy of this fully functional complex yielded a resolution of about $4 \AA$. From the observed populations of the complex, the remodeler was found to bind at the nucleosome preferably in different locations on DNA, i.e. either on superhelical location SHL 2, on SHL 6 and simultaneously on both SHL 2 and SHL 6 [22]. We take this basic information as key ingredients to be reflected in our model, which therefore should be able to describe a basic chromatin remodeler without any further additional recognition domains.

Although precise dynamical information cannot directly be inferred from the cryoEM data, the following facts can be considered as established, also in conjunction with 
earlier work: (i) the remodeler structure consists of two lobes (also called 'cores', in fact presumably properly folded protein domains), which form multiple contact surfaces with both DNA turns; (ii) upon remodeling, the remodeler injects twist defects into the DNA turns which are expelled at the end contact of the remodeler domains with the DNA; (iii) the remodeler thus performs a spatially restricted rotation at the nucleosome turns: its full rotation around the DNA is impeded by the presence of the histone core. This limited rotary motion of the remodeler is a remainder of its helicase-like nature: a helicase would fully rotate around a single DNA double-strand during its linear motion along the DNA [25]. This rotation is represented in some models of helicases, but not by the Brownian dimer model. In our case, this neglect is justified because of the specific arrangement of the remodeler at the nucleosome. Figure 1 (left) displays a sketch of the remodeler profile at the DNA turns around the nucleosome.
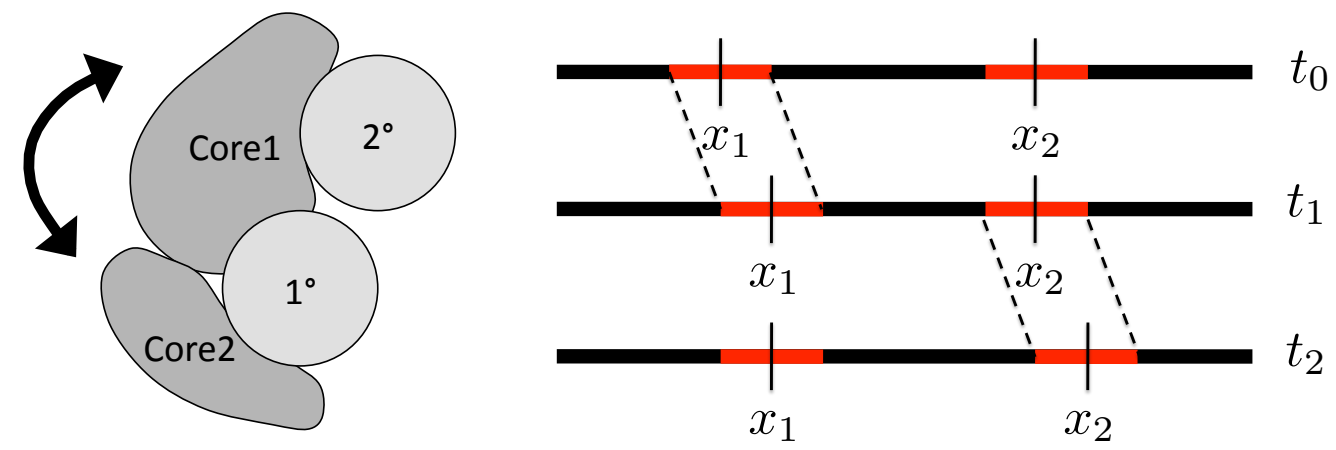

Figure 1. Two representations of the action of the remodeler on nucleosomal DNA. Left: side-view of the engagement of the two remodeler lobes with the two turns of DNA, following $[22,23]$. Right: Mapping of the remodeler-DNA contacts on the two remodeler footprints denoted by coordinates along the DNA centered at $x_{i}, i=1,2$. Each black bar represents the length of DNA along the nucleosome, with the red bars indicating the footprints (contact surfaces) of the remodeler along the DNA. Top bar: initial configuration at time $t_{0}$. Middle bar: after a first step step $t_{1}$, the left footprint has moved to the right while the other is still unchanged. Lower bar: at time $t_{2}$ the right footprint has also moved and the displacement step has been completed.

Turning to modeling this behaviour, condition (iii) allows to neglect the rotary motion of the remodeler; conditions (i) and (ii) then allow to restrict the dynamics to the footprints of the two lobes on DNA, which we denote as in $[21,26]$ as $x_{1}$ and $x_{2}$. In the course of the remodeler action, footprint $x_{1}$ is first shifted towards location $x_{2}$ via twist defect injection; the displacement of footprint $x_{2}$ follows in due course leading to twist defect ejection. The DNA length $x \equiv x_{1}-x_{2}-x_{0}$ where $x_{0}$ is the equilibrium extension of the DNA around the nucleosome thus relaxes after one remodeler step which is typically $1-2$ bp large $[27,28,29]$; the variable $x$ is therefore small, irrespective of the location of $x_{1}$ and $x_{2}$ along the internucleosomal DNA. This mapping of the displacement dynamics is illustrated in Figure 1 (right). The motion of the footprints thus is indeed akin to an inchworm motion of DNA around the histone octamer [23]. 
Modeling the inchworm. This inchworm motion can be rationalized in terms of molecular motor models, for which there are two philosophies. The most common one relies on Brownian ratchet models, where the key ingredient is the ratchet potential, a potential with a sawtooth-profile, which controls motor motion in conjunction with the ATP-consumption cycle. For chromatin remodelers such models have been developed for the case of a remodeler from the ISWI family which is capable to position nucleosomes on DNA; this could be demonstrated experimentally in single-molecule positioning assays $[4,5]$. For the present case in which the motion is that of a motor with two footprints, a ratchet model has been discussed in the literature [33]. The other modeling philosophy relies on active Brownian dimer models [21, 26], which is the approach we follow here.

In the overdamped case which we assume in the following, it is given by coupled Langevin equations for the two footprint coordinates $x_{i}$,

$$
\gamma_{i}(x) \dot{x}_{i}(t)=-\partial_{i} U(x)+g_{i}(x) \xi_{i}(t)
$$

where $\xi_{i}(t)$ is a Gaussian noise with zero mean $\left\langle\xi_{i}(t)\right\rangle=0$ and variance $\left\langle\xi_{i}(t) \xi_{j}(t)\right\rangle=$ $\delta_{i j} \delta\left(t-t^{\prime}\right)$. Three key factors appear in this equation. The first is a spatially-dependent friction term $\gamma_{i}(x) \geq 0$ which qualitatively models the remodeler-nucleosome interaction. The second term is the potential $U(x)$ which we ascribe to the interaction of the remodeler domains between the two footprints. Both depend on the relative coordinate $x$ introduced before. Finally, the factor $g_{i}(x)$ describes the noise correlations. It is given by

$$
g_{i}(x) \equiv \sqrt{2 \gamma_{i}(x) k_{B} T+A_{i}}
$$

where the factor $A_{i}$ describes the contribution of non-equilibrium noise due to ATPconsumption.

This active Brownian dimer model is obviously highly coarse grained, since the nonequilibrium ATP-dependent driving force is not associated with particular configurations of the remodeling-based ATP cycle (we comment on this further in the discussion). Crucial in these kinds of models is the asymmetry in the spatially-dependent coupling of the motor to the twist-stretched DNA: neglecting the spatial dependence destroys the propagation mode in the model.

The dynamics of the two footprints can be decoupled into a center of mass motion and the relative coordinate. Due to the dependence of $\gamma_{i}(x)$ and $U(x)$ on only the relative coordinate $x$ the equation of the center of mass coordinate $x_{c m}$ is a function of the relative coordinate and the noise, while the equation for the relative coordinate is independent from the center of mass coordinate. The dynamics of the latter can be cast into a Fokker-Planck equation for the probability distribution of the relative coordinate $P(x, t)$ which reads as [26]

$$
\partial_{t} P(x, t)=-\partial_{x}[a(x) P(x, t)]+\frac{1}{2} \partial_{x}\left[b(x) \partial_{x}[b(x) P(x, t)]\right]
$$


which has a stationary solution given by

$$
p(x)=\frac{N}{b(x)} \exp \int^{x} d y \frac{2 a(y)}{b^{2}(y)}
$$

where $N$ is the normalization factor. In the following, based on the model assumptions, we choose for $\gamma_{i}(x)$ the following expressions. Shifting the $x$-dependence into one of the footprint coordinates, we define $\gamma_{1}=\gamma_{0}$ and $\gamma_{2}=\gamma_{0}+\gamma x$, similar to the choice in [21]. The zeroth-order term $\gamma_{0}$ can be understood as the translational friction term deriving from thermal nucleosome displacement [30,31]. For the present case, the detailed form of the $x$-dependence in $\gamma_{2}$ is irrelevant, since $x$ is always small in our case. We now exploit this case, which was not done in [26], where other choices were studied by numerical means. The smallness of $x$ allows us to obtain analytical results for the model. Finally, it suffices to approximate the domain coupling $U(x)$ by a harmonic potential with an effective elastic constant $\kappa, U(x)=\frac{1}{2} \kappa x^{2}$.

In our case the coefficients in the Fokker-Planck equation are given by the expressions

$$
a(x)=-\left(\frac{1}{\gamma_{0}}+\frac{1}{\gamma_{0}+\gamma x}\right) \kappa x-\frac{k_{B} T}{2} \frac{\gamma}{\left(\gamma_{0}+\gamma x\right)^{2}},
$$

where the $T$-dependent term has its its origin in the noise term of the Langevin equation, eq.(2) which has both thermal and 'active' contributions, and

$$
b^{2}(x)=2 k_{B} T\left(\frac{1}{\gamma_{0}}+\frac{1}{\gamma_{0}+\gamma x}\right)+\frac{A}{\left(\gamma_{0}+\gamma x\right)^{2}} .
$$

where we have set $A_{1}=0$ and $A_{2}=A$. In the limit $\gamma \ll \gamma_{0}$, the coefficients simplify to

$$
a(x) \approx-\frac{2}{\gamma_{0}} \kappa x-\frac{k_{B} T}{2} \frac{\gamma}{\gamma_{0}^{2}} \approx-\frac{2}{\gamma_{0}} \kappa x,
$$

where we neglect the last, temperature-dependent term, as it is small compared to the first term. Further, we have

$$
b^{2}(x) \approx \frac{4 k_{B} T}{\gamma_{0}}+\frac{A}{\gamma_{0}^{2}}
$$

In this case, the probability distribution reduces to a simple Gaussian given by

$$
p(x)=\frac{1}{\sqrt{2 \pi \sigma^{2}}} \exp -\frac{(x-\mu)^{2}}{2 \sigma^{2}} .
$$


with zero mean value, $\mu=0$, and the variance

$$
\sigma^{2}=\frac{A+4 k_{B} T \gamma_{0}}{4 \gamma_{0} \kappa}
$$

Note that for $A=0, p(x)$ is the equilibrium Boltzmann distribution. From this distribution we can then compute the average inchworm velocity using the expression derived in Ref. [26]. The authors have given the exact expression

$$
\begin{aligned}
\left\langle\dot{x}_{c m}\right\rangle= & -\frac{1}{2}\left\langle\Delta \gamma_{i}(x) \partial_{x} U(x)\right\rangle+ \\
& \frac{k_{B} T}{2}\left\langle\partial_{x} \Delta \gamma_{i}(x)\right\rangle+\sum_{i}(-1)^{i} \frac{A_{i}}{4}\left\langle\frac{\partial_{x} \gamma_{i}(x)}{\gamma_{i}(x)^{3}}\right\rangle
\end{aligned}
$$

with the averages $\langle\cdot\rangle$ to be taken with respect to $p(x)$ and the definition

$$
\Delta \gamma_{i}(x)=\gamma_{1}^{-1}(x)-\gamma_{2}^{-1}(x)
$$

In our case the expression reduces to

$$
\left\langle\dot{x}_{c m}\right\rangle=-\frac{\gamma}{2 \gamma_{0}^{2}}\left\langle\kappa x^{2}\right\rangle+\frac{k_{B} T \gamma}{2 \gamma_{0}^{2}}+\frac{A}{4} \frac{\gamma}{\gamma_{0}^{3}},
$$

whereby the two last terms follow from the averages to be performed on the approximate equation

$$
\Delta \gamma_{i}(x)=\gamma_{1}^{-1}(x)-\gamma_{2}^{-1}(x)=\gamma_{0}^{-1}-\left(\gamma_{0}+\gamma x\right)^{-1} \approx \frac{\gamma}{\gamma_{0}} x
$$

The remaining average over $\left\langle x^{2}\right\rangle$ produces the final result

$$
\left\langle\dot{x}_{c m}\right\rangle=-\frac{A}{8} \frac{\gamma}{\gamma_{0}^{3}}-\frac{k_{B} T}{2} \frac{\gamma}{\gamma_{0}^{2}}+\frac{k_{B} T}{2} \frac{\gamma}{\gamma_{0}^{2}}+\frac{A}{4} \frac{\gamma}{\gamma_{0}^{3}}=\frac{A}{8} \frac{\gamma}{\gamma_{0}^{3}} .
$$

Thus, performing the average the $T$-dependent terms - which are themselves small within our approximation - cancel each other out exactly, as they should on general grounds: in the absence of active driving, the average inchworm velocity must be zero [26]. The final result for the inchworm velocity depends only on both the active noise $A$ and the stretch-dependent driving via $\gamma$ and $\gamma_{0}$, but neither on the harmonic potential $\kappa$ nor temperature. These dependencies enter back into the final result when going to higher orders in our approximation. Keeping the next order in the displacement $x$ leads to a skewed distribution for the displacement, $p(x)$, as found numerically in [26]. A finite mean of the distribution, e.g. with a positive value $\mu>0$, goes hand in hand with its skewness towards negative values of $x$ in order to allow for the cancellation of temperature-dependent terms in the limit $A \rightarrow 0$. 
One can compare the putative inchworm motion with experiments on artificial motor constructs, as done for the remodeler RSC by Sirinakis et al. [32]. In that paper the maximum velocity of the remodeler is obtained from the location of the maximum of the probability distribution of observed velocities as a function of ATP consumption $[A T P]$, which shows a weaker logarithmic dependence on driving, $v_{\max } \sim \ln [A T P]$. In the experiments of ref. [32], the motor was employed on a linearized DNA sequence under external load; the propagation of the DNA was then actually by the formation of loops. Clearly, this is a different regime of helicase action, since for the remodeler acting on the nucleosome, the external load is given by the DNA itself.

Discussion. In this paper we have formulated a coarse-grained minimal model of a chromatin remodeler which combines three elements: i) structural insights into the build-up of a 'minimal' chromatin remodeling module; ii) a mapping to the class of active Brownian dimer models introduced several years ago for simple helicases; iii) an exact solution of the model which furthermore exploits the fact that the twist-stretch coupling is always small in the present case. The model allows to rationalize the notion of inchworm motion in the context of remodelers and makes a simple prediction of the expected inchworm velocity as a function of active driving and the twist-stretch coupling. Higher-order corrections to our analytic limit can be identified with the strength of the remodeler lobe coupling and temperature.

Obviously, the model we present is extremely reduced. It can, however, easily be extended in both more microscopic and macroscopic directions: in order to include detail of nucleosome structure and coupling it to the proper ATP-cycle, modeling approaches such as cited before in terms of ratchet models [33], or very recent novel active models [34] can be applied. In this way, more information about the force-generation leading to the inchworm motion would be built in. On the other hand, it would also be of high interest to take the coarse-grained Brownian dimer for the confrontation with experiments: measuring the inchworm velocity as a function of the active driving force would allow to estimate the friction parameters in eq.(15). A further outlook is that the Brownian dimer model can be taken as a starting point for the derivation of chromatin hydrodynamics models beyond phenomenology [10].

Acknowledgement. We thank Dr. Giovanni Brandani and Dr. Andrew Flaus

for helpful discussions on chromatin remodelers. RB also is grateful for discussions on molecular motor models with Prof. Christian Maes.

\section{References}

[1] D. Chowdhury. Phys. Rep. 529, 1-197 (2013)

[2] P. Romanczuk, M. Bär, W. Ebeling, B. Lindner and L. Schimansky-Geier, Eur. Phys. J. Special Topics, 1-162 (2012)

[3] T. Chou, Phys. Rev. Lett. 99, 058105 (2007)

[4] Y. Vandecan and R. Blossey, Phys. Rev. E 85, 061920 (2012)

[5] Y. Vandecan and R. Blossey, Phys. Rev. E 88, 012728 (2013) 
[6] K.H. Iwasa and A.M. Florescu, J. Stat. Mech. Theor. Exp., 054030 (2016)

[7] V.B. Teif, K. Rippe, Nucl. Acids Res. 37, 5641-5655 (2009)

[8] R. Padinhateeri and J.F. Marko, Proc. Natl. Acad. Sci 108, 7799 (2011)

[9] A.M. Florescu, H. Schiessel and R. Blossey, Phys. Rev. Lett. 109, 118103 (2012)

[10] R. Bruinsma, A.Y. Grosberg, Y. Rabin and A. Zidovska. Biophys. J. 106, 1871-1881 (2014)

[11] J.N. McKnight, T. Tsukiyama and G. D. Bowman. Genome Res. 26, 693-704 (2016)

[12] I.M. Nodelman, F. Bleichert, A. Patel, R. Ren, K.C. Horvath, J.M. Berger and G.D. Bowman. Mol. Cell. 65, 447-459 (2017)

[13] J. Winger and G. D. Bowman. J. Mol. Bio. 429, 808-822 (2017)

[14] R. Sundaramoorthy et al.. eLife 6, e22510 (2017)

[15] L. Farnung, S.M. Vos, C. Wigge and P. Cramer. Nature 550, 539-542 (2017)

[16] C.R. Clapier and B. R. Cairns. Annu. Rev. Biochem. 78, 273-304 (2009)

[17] C.L. Smith and C.L. Peterson. Current Topics in Developmental Biology 65, 115-148 (2004).

[18] W.K.M. Lai and B.F. Pugh. Nature Rev. Mol. Cell. Biol. 18, 548-562 (2017)

[19] R. Blossey. Chromatin: Structure, Dynamics, Regulation CRC Press Boca Raton, USA (2017)

[20] R. Kumar, D.-Q. Li, S. Müller and S. Knapp. Oncogene 35, 4423-4436 (2016)

[21] K.V. Kumar, S. Ramaswamy and M. Rao. Phys. Rev. E 77, 020102 (2008)

[22] X. Liu, M. Li, X. Xia, X. Li and Z. Chen. Nature 544, 440-445 (2017)

[23] D.A. Wigley and G.D. Bowman. Nature Struct. Mol. Biol. 24, 498-500 (2017)

[24] P.T. Lowary and J. Widom. J. Mol. Biol. 276, 19-42 (1996)

[25] C. Doering, B. Ermentrout and G. Oster. Biophys. J. 69, 2256-2267 (1995)

[26] A. Baule, K.V. Kumar and S. Ramaswamy. J. Stat. Mech. P1108 (2008)

[27] S. Deindl et al. Cell 152, 442-452 (2013)

[28] B.T. Harada et al. eLife 5, e10051 (2016)

[29] G.B. Brandani and S. Takada, PLoS Comp. Bio. 14, e1006512 (2018)

[30] I.M. Kulic and H. Schiessel. Chromatin Dynamics: Nucleosomes go Mobile through Twist Defects Phys. Rev. Lett. 91, 148103 (2003)

[31] H. Schiessel. Biophysics for Beginners. PanStanford (2013)

[32] G. Sirinakis, C.R. Clapier, Y. Gao, R. Viswanathan, B.R. Cairns and Y. Zhang, EMBO J. 30, 2364-2372 (2011)

[33] Y.A. Makhnovskii, V.M. Rozenbaum, S.-Y. Sheu, D.-Y. Yang, L.I. Trakhtenberg and S.-H. Lin. J. Chem. Phys. 140, 214108 (2014)

[34] T. Demaerel and C. Maes. Phys. Rev. E 97, 032604 (2018) 